\title{
CONTROL ALGORITHM DEVELOPMENT FOR MOBILE FSO NODE
} ALIGNMENT

\author{
Dayong Zhou, Hazem H. Refai, School of Electrical \& Computer Engineering, University of \\ Oklahoma, Tulsa, Oklahoma \\ Peter G. LoPresti, Department of Electrical Engineering, University of Tulsa, Tulsa, Oklahoma \\ Mohammed Atiquzzaman, School of Computer Science, University of Oklahoma, Norman, Oklahoma
}

\begin{abstract}
Since the need for high alignment accuracy of the transmitter and receiver to construct a viable connection is a primary challenge for mobile freespace optical (FSO) links, developing an efficient transmitter control system for acquisition and tracking is an urgent demand. This paper investigates a novel control algorithm within different transmitter designs in FSO mobile networks. The fiber bundles are utilized so that the beam deflection properties of a lens can be exploited to affect beam steering. After acquiring and processing the data from GPS, fisheye system as well as the receiver, the adaptive control system would help tracking the mobile receiver and providing optimum coverage over the target area. Initial analysis of the design indicates refinements in design and performance required to develop a practical control system.
\end{abstract}

\section{Introduction}

Free-space optical (FSO) communication can provide a way to establish high-bandwidth links for a variety of applications, including inter-satellite communication, airborne internet, and inter-building communication in urban settings: the latter is commercially available for a range of distances and system requirements [1-3]. FSO has proven especially useful as a complement to radio frequency (RF) networks, providing a higher bandwidth channel for moving large data volumes and providing connectivity in conditions unfavorable for RF communication [4-6].

In order to acquire, track and maintain a freespace optical link between mobile platforms experiencing misalignment due to movement and atmospheric turbulence requires a different approach than traditional free-space optical transceivers. Recently, a fiber-bundle approach for beam steering at the transmitter was proposed and investigated that allowed tracking of the receiver without the use of mechanical devices. A complimentary receiver design was developed to improve the angular and translational misalignment that could be tolerated and still potentially maintain a viable link. Despite these developments, a control system is still required to point the transmitter and receiver in the general direction of each other and to provide fine adjustments to both acquire and maintain the link connectivity.

In this paper, a control algorithm is proposed and evaluated for accomplishing acquisition and tracking. The algorithm assumes the existence of a master control system for realizing course alignment of the mobile platform, in this case a balloon or blimp. The algorithm takes GPS and control data from the course alignment system to estimate the relative orientation of the node transceivers. This data is then combined with data from a guide beam system utilizing standard quadrant detectors to assess several factors that influence control decisions. These factors include the transmitter design implemented at the node, the likelihood of completing the link with or without minor adjustments in the transmitter power and beam divergence, and foreknowledge of the receiver design. Control actions at the fine optical level or the course mechanical level are then dictated and acted upon. Initial evaluation of the design is performed and indicates refinements in design and performance required to develop a practical control system.

\section{Background}

Recent disastrous events, most notably $9 / 11$ and several major hurricanes have highlighted the vulnerability of the current telecommunications infrastructure to man-made and natural catastrophes. Overwhelming destruction and the subsequent loss of services significantly disrupted or destroyed critical resources and subsequently cause key 
communications systems to fail. These failures highlighted the vulnerability of the telecommunications infrastructure and clearly identified the need for a rapidly deployable communications system to be used in the disasteraffected area to support the rescue and recovery efforts. With the destruction of cellular towers, underground coax cables and optical fibers, and telecommunications switches and offices, communication when it is much needed comes to a halt. Lack of communication during disaster recovery efforts can result in a delayed and inefficient rescue and recovery effort that has the potential to increase the probability of loss of lives.

The focus of our ongoing work is the investigation and implementation of a disaster area wireless network (DAWN) using a hybrid free space optical and RF communication systems to provide communication immediately after a nature or manmade event. FSO is capable of providing high bandwidth, secure, and interference hardened communication, while RF wireless accommodates terminal mobility in the era of portable and pervasive computing. DAWN technology uses helium-filled balloons, securely tethered to the ground, or rapidly deployable towers, to rapidly launch a wireless mesh network and establish communication over the affected area. It uses WiFi technology and widely available WiFi-based applications to provide voice, video, and data communication capabilities to mobile terminals on the ground. It also uses FSO technology to establish an optical backbone between balloons for aggregating and hauling traffic generated in the RF network to the Internet. Connection to the Internet is established by either an FSO terminal or an optical fiber. A conceptual diagram of a DAWN system using two mobile nodes is shown in Figure 1. A propulsion system in manual mode allows the system's ground operator to make directional adjustments and manipulate flight from one GPS waypoint to another. Data are transmitted to the fixed network via FSO, WiFi, or Cellular network, respectively.

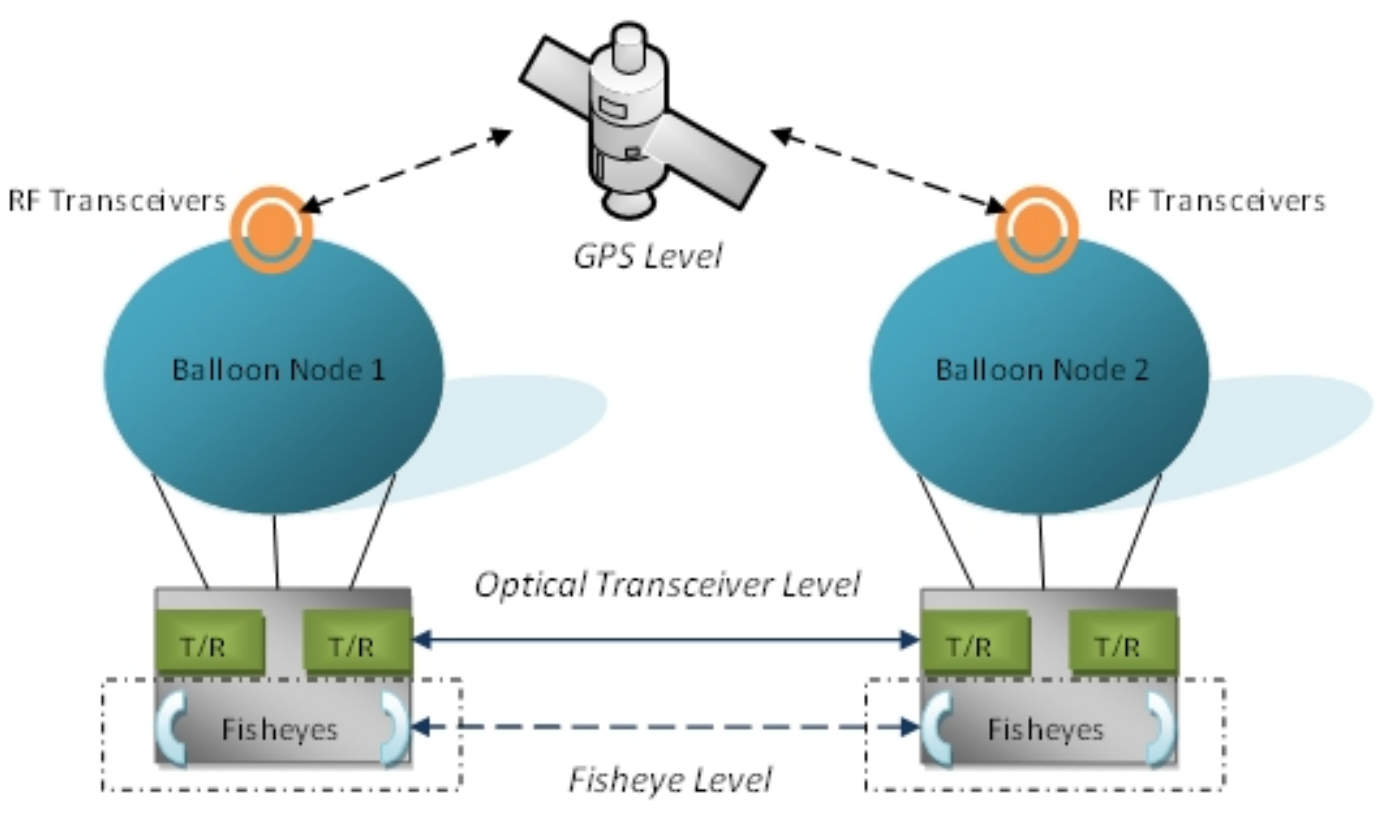

Figure 1. System Overview

On the basis of expected accuracy, the FSO alignment system can be divided into three levels. The first level is the GPS level, which collects and processes the location information for each node from GPS satellites. The GPS data for potential target nodes is either obtained from a GPS data repository (satellite or other stable node fixture) or from exchanges between the nodes over an RF management channel. Knowledge of the node locations enables the Balloon Control System (BCS) to initially adjust the balloon orientations to roughly align the respective transceivers. At the second level, an optical alignment 
system based on a standard fisheye lens and quadrant detector, simply referred to as the fisheye system, provides a method to obtain the data required to make finer alignment modifications. With an over 180 degrees field of view, fisheye lens has the ability to capture incoming beams from a larger range of directions than optical transceiver lenses. The electronic quadrant detector guides the BCS and Optical Control System (OCS) to correct alignment. The third level of alignment is the optical transceiver level which directs the last step of alignment through beam control. By adjusting the beam divergence and achieving optical beam steering in a small physical space, the link can exhibit a greater tolerance for alignment error by providing a sufficient link budget over a larger spatial volume. In this work, we assume optical fiber bundles combined with appropriate optical components choices are used [7]. This design is able to generate continuous coverage of a target area without the weight and stabilization issues of mechanical systems.

The proposed transceiver system is depicted in Figure 2. A signal to be transmitted is directed to one (or more than one) of seven fibers arranged in a hexagonal geometry at the transmitter. A signal directed to the central fiber propagates along the central axis of the transmitter. If the transceivers are misaligned, the signal can be directed to a fiber away from the axis. Light from this fiber is deflected at an angle from the axis by the action of the lens, effectively steering the beam through that angle. The distance from the fibers to the transmitting lens is varied to control the beam divergence while still satisfying a certain power budget. Light is collected at the receiving end and appropriately processed to recover the transmitted signal.

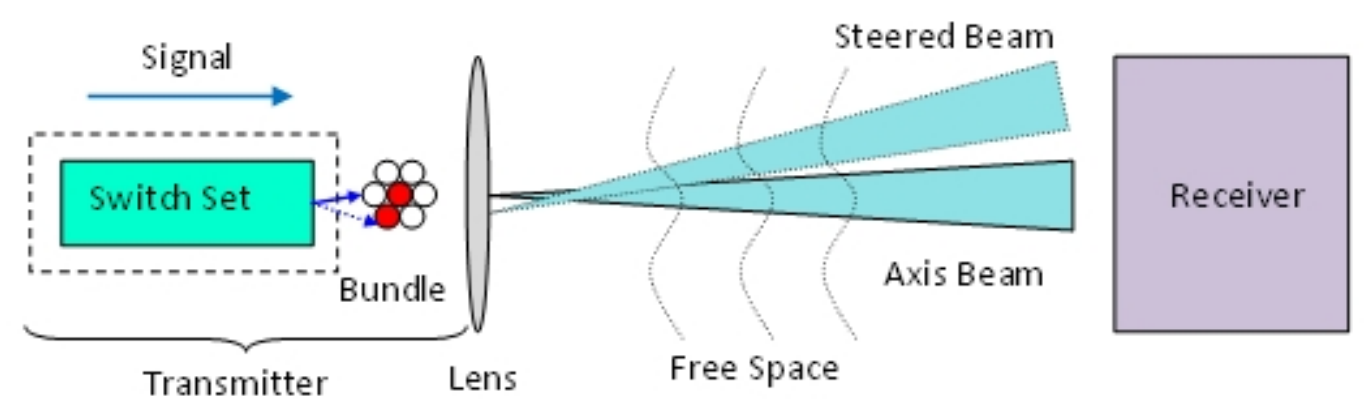

Figure 2. Optical Transceiver System

According to the optical components in use, the relevant designs are organized into three basic groups: "All Couplers" designs, "All Switches" designs and "Couplers/Switches Hybrid" designs. "All Couplers" designs distribute optical power over all of the seven fiber outputs, meaning that the maximum beam coverage can be achieved at the target area without switching between fibers, preventing switching time from becoming an issue. Figure 3 illustrates one "All Couplers" design, which contains a single power source working with one $1 \times 8$ coupler. As Figure 4 shows, "All Switches" designs concentrates the optical power to only one fiber output so that the highest power density can be achieved at the receiver and there is no unnecessary power loss to the other fiber outputs that do not connect with the receiver. The benefits that are achieved are a result of the switches' ability to steer the signal. One of the "Couplers/Switches Hybrid" designs in Figure 5 consists of a single power source, one $1 \times 2$ switch and two $1 \times 4$ couplers. This kind of design is expected to combine the larger beam coverage from the "All Couplers" design and less power loss from the "All Switches" Design.

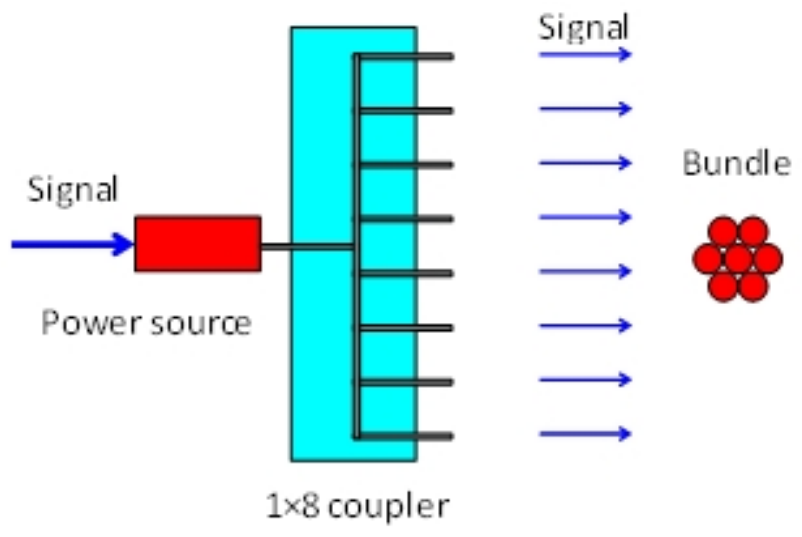

Figure 3. "All Couplers" Design 


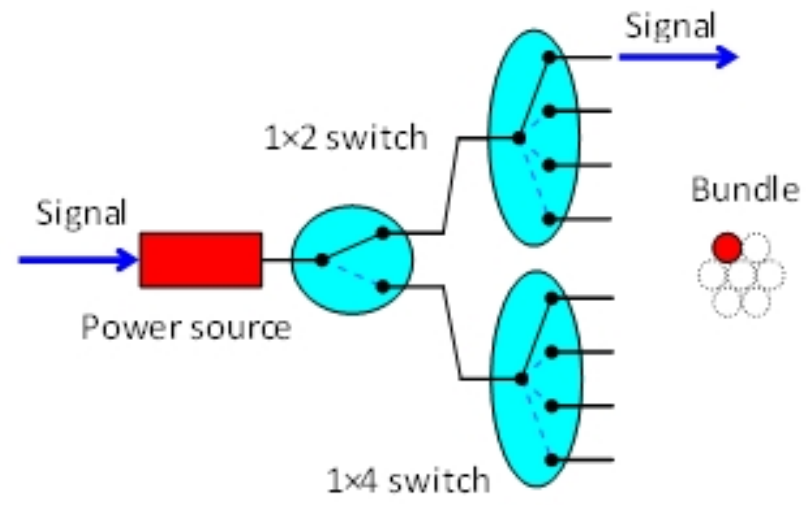

Figure 4. "All Switches" Design

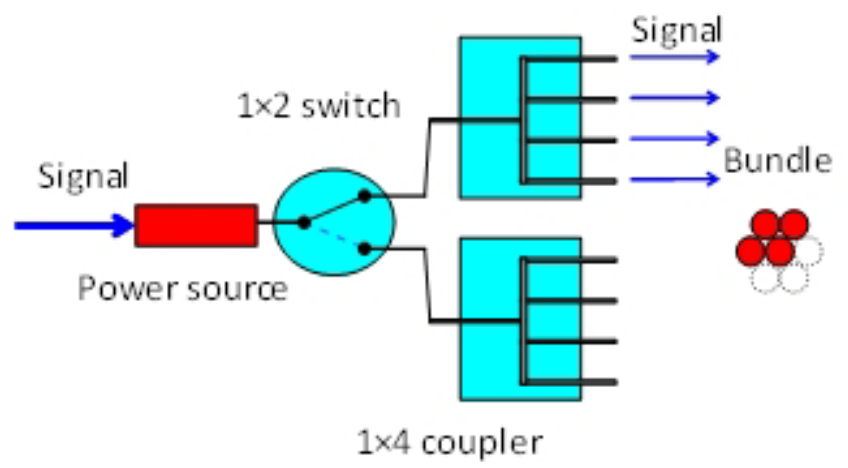

Figure 5. "Hybrid" Design

Prior investigations of these designs [7] demonstrated that, in general, a coupler and switch combination provided the best compromise of power budget and tracking time. Some applications, however, would be best served by an all-coupler or all-switch design, depending on whether power or switching speed is the dominant factor.

\section{Control Algorithm}

The optical control algorithm contains three phases in sequence: System Starting, Finer Correction and T-R Link Checking. Figure 6 shows the optical alignment control flow chart.

\section{Phase A: System Starting}

At the System Starting phase, the distance $L$ between the two mobile nodes is calculated with the information collected from the GPS location data for each balloon, and then an optimal fiber-lens distance at the receiver is selected that optimizes the likelihood of a successful connection based on the value $L$, the specifics of the particular transmitter design and a particular link acquisition strategy. While these adjustments are taking place at the optical transceiver level, the BCS takes action to adjust the orientation of the balloon (or other relevant platform) to achieve a basic level of alignment between the transceivers. The control system must then wait for BCS to stabilize on a final position before continuing the alignment process.

\section{Phase B: Finer Correction}

For the second phase, the fisheye systems are utilized to make finer corrections. The first step is to get the magnitude and direction of error as interpreted by the quadrant detector behind the fisheye lens. This action is taken for both nodes in the link, each node acting only on its own information. For reference for this discussion, the plane of movement parallel to the ground is considered the $x-y$ plane, and the direction perpendicular to the ground is the $\mathrm{z}$ axis.

In the second step, the errors $\Delta x, \Delta y$ and $\Delta z$, for the $\mathrm{x}, \mathrm{y}$, and $\mathrm{z}$ directions respectively, are calculated and fed to the appropriate controllers. The balloon adjustment is limited by the control components, and for this discussion movement is possible only within the $\mathrm{x}-\mathrm{y}$ plane, so only the $\Delta \mathrm{x}$ and $\Delta \mathrm{y}$ data is needed for BCS. Since the OCS has the ability to make adjustments in all directions, the OCS requires $\Delta \mathrm{x}, \Delta \mathrm{y}$ and $\Delta \mathrm{z}$ information.

In the third step, the two control systems determine whether control actions will be executed by the respective system, and what the appropriate action should be. To determine which control system should take action, control boundaries are constructed that divide the control space. Two factors determine these boundaries: the dynamic range of the optical transmitter (switching angle, optical power, and optical divergence) and foreknowledge of which actions are potentially beneficial and which actions are selfdefeating. If the error calculation indicates that the corrective action required is outside the capabilities of the OCS, then the BCS is directed to act in an effort to achieve a better initial alignment condition. If the error calculation indicates that the corrective action is within the capabilities of the OCS, then action is requested of that system. 


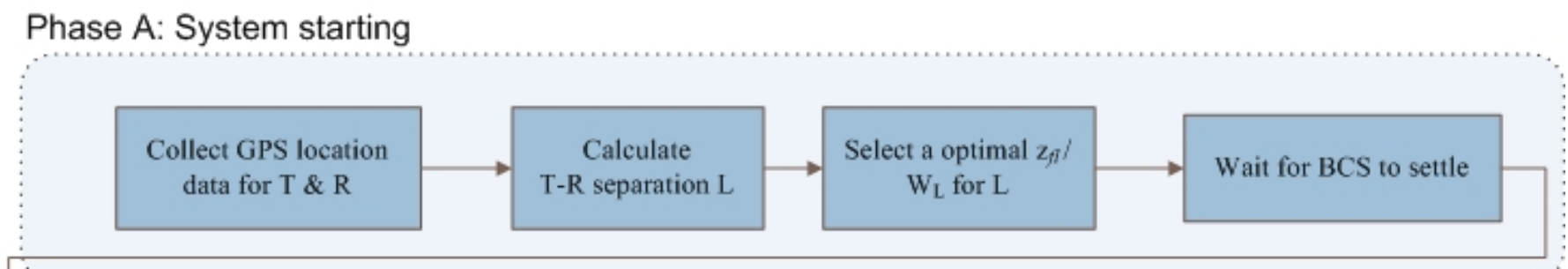

\section{Phase B: Finer correction}

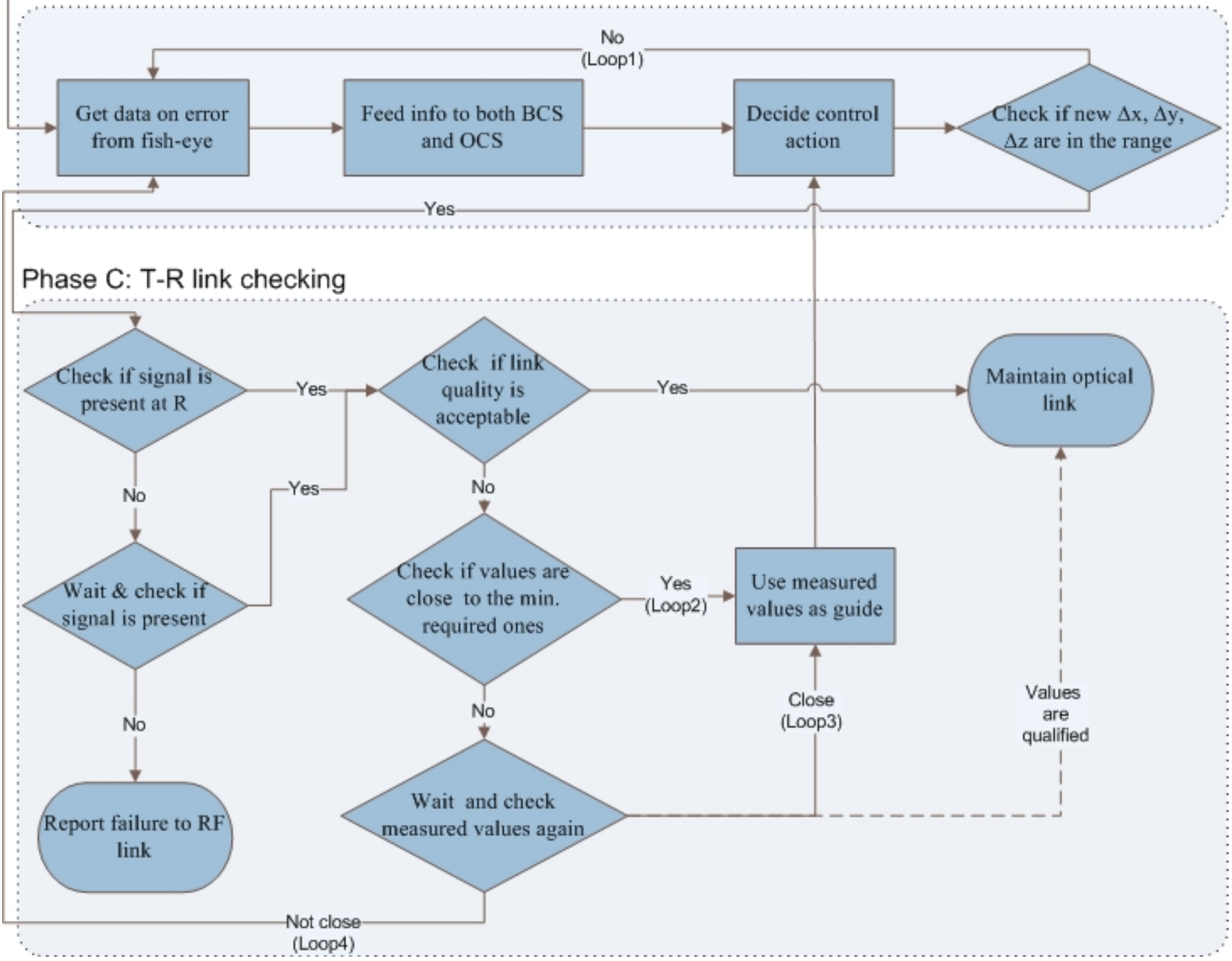

\section{Figure 6. Control Algorithm Flow Chart}

The final step is to check the fisheye system again to determine whether the new $\Delta \mathrm{x}, \Delta \mathrm{y}$ and $\Delta \mathrm{z}$ values are within an acceptable range for transceivers. Here, an acceptable range is defined as a misalignment that is within the tolerance of the link design and for which a successful link is therefore likely to occur. If the misalignment is tolerable, then the system can proceed to the next phase; if not, the system updates the error information through quadrant detector and returns to the first step in the cycle. 


\section{Phase C: T-R Link Checking}

The first step in this cycle is to determine whether a viable link is available for transmission.

If a signal is present at the receiver, then check the link quality by measuring the received power $\mathrm{P}_{\mathrm{r}}$, the bit error rate (BER) and the optical signal-noise ratio (OSNR). If the quality is acceptable, use the fisheye system and the procedure in Phase B to maintain the FSO link. Additional steps may be added to provide a predictive capability, where successive position readings, along with any motion related data provided by the GPS unit, are used to determine control actions in advance, with the goal of minimizing gaps in the link up-time.

If the link quality is poor, determine if the measured performance quantities are close enough (within some predetermined limit) to the minimum required values. If the performance is close, indicating that only minor adjustments are required to improve the link operation, use the measured values as guide to perform fine correction at OCS level. If the performance is too degraded, wait a short time and measure the performance again before taking any large-scale corrective action. The purpose of this short delay is to prevent major corrective action if the degradation in performance is caused by some temporary obstruction or event rather than an actual system misalignment. If the new measured values are still not acceptable return to the Finer Correction phase to implement a more significant control action as needed.

If signal is not present at receiver at all, wait for a predetermined time interval and then check again to see if a signal is present. If the signal now appears, follow the procedure just describe. If there is still no signal, and generate a link failure report to network so that data can be sent over a different path until the optical link can be reconnected. Note that we assume that the fine correction, using the guide laser and the fisheye system, would at least provide sufficient alignment to detect the presence of the transmitted beam energy, if not recover the signal. Therefore, it is acceptable to report a link failure when repeated attempts to detect the signal power fail.

\section{Laser Beam Adjustment at OCS}

At the Finer Correction phase, the details of the beam adjustments performed by the OCS depend, necessarily, on which of the transmitter designs have been implemented. Details of the process for each of the previously described designs are as follows.

Since the "All Couplers" design uses all of the fibers simultaneously, the main question is whether or not the existing misalignment places the target within or near the coverage area for which the ability to achieve a viable link is projected. If the target is within the coverage area, then no further control action is required, and the process can proceed to the link-checking phase. If the target is close to the edge of the beam coverage area, then the appropriate control action is to adjust $\mathrm{P}_{\mathrm{t}}$ and/or $\mathrm{z}_{f l}$ to increase the coverage area slightly. If this is done properly, the new coverage area will now include the target location, and the system can now focus on link acquisition. The definition of close depends on the limits on the control system's dynamic range and the current state of the transmitter parameters. If the target position is deemed to be too far from the coverage area for acquisition by only the OCS adjustments, then control actions are requested from the BCS to perform corrections to the balloon orientation. The flowchart for this process is shown in Figure 7. 


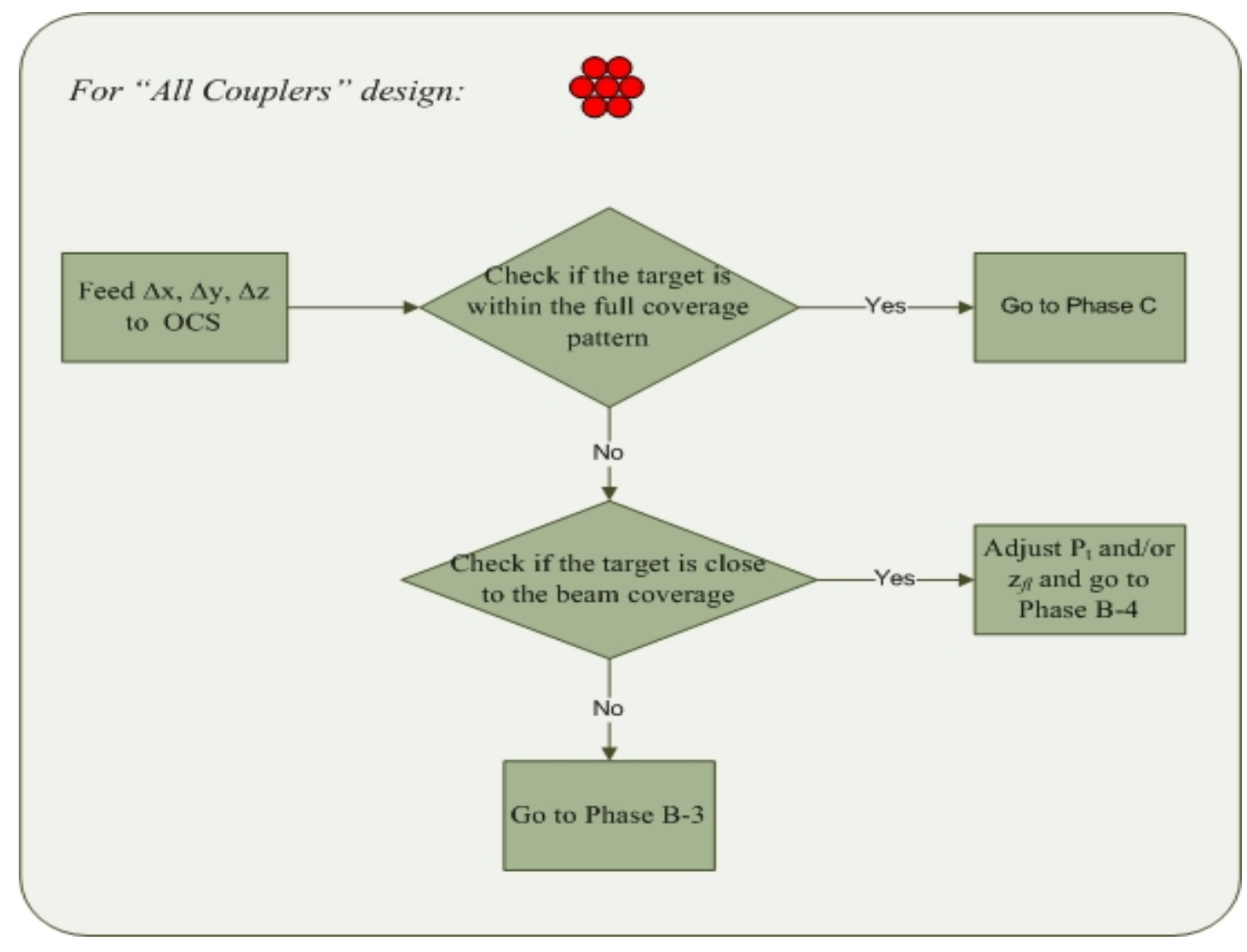

Figure 7. Control Flow for "All Couplers" Design

For the "All Switches" design, the goal is twofold: choose the fiber most likely to direct the signal toward the target location, and adjust the beam divergence and power as necessary to insure a connection. Therefore, the first step is to check if the target is within the current coverage pattern as defined by which fiber in the bundle is currently lit. If the target is so positioned, then no further control action is required, and the process can proceed to the linkchecking phase. Some adjustment of the beam power and divergence may be required if the target location is located at the very edge of the coverage area, since switching to a different fiber will only put the target on the edge of that fiber's coverage area. If the target is not within the current coverage area, a quick calculation determines which fiber of the transmitter bundle would cover the target's location. This calculation includes the potential coverage pattern of each fiber, as determined by the outer limits on beam divergence and transmitter power. If the target lies within the coverage area of one of the fibers in the bundle, then the optical power is switched to that fiber and necessary adjustments to the transmitted beam are made. If the target is outside of the potential coverage area, then control actions are requested from the BCS. A flowchart of this process is shown in Figure 8. 


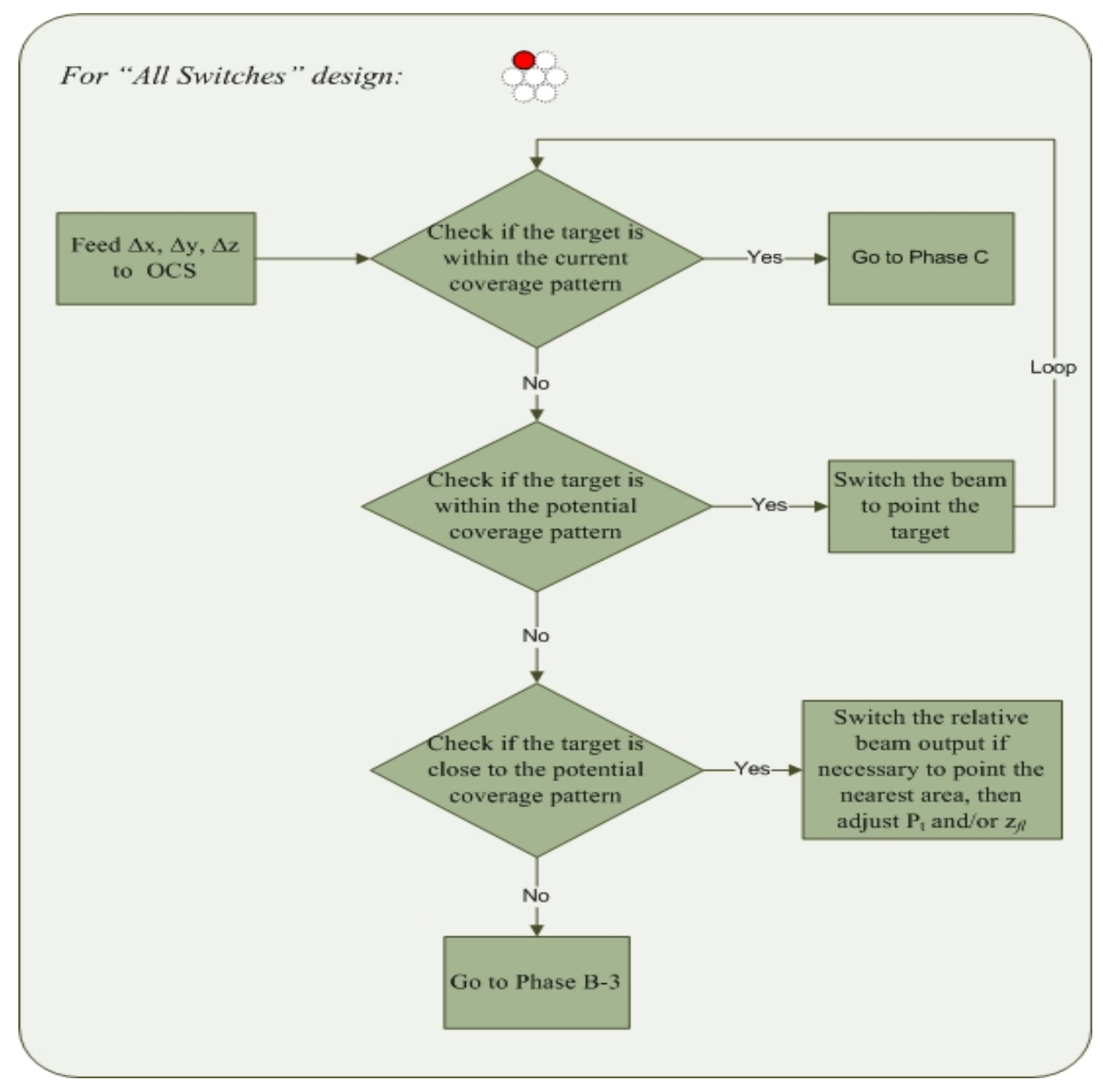

Figure 8. Control Flow for "All Switches" Design

For the "Hybrid" design, the control process is necessarily a hybrid of the processes for the other two design choices. The first step is to check if the target is within the current coverage pattern determined by which fibers are lit and the optical beam parameters currently in use. If so, then no further control actions are necessary and the process can proceed to the linkchecking phase. If the target is not in the current coverage pattern, the next choice is to determine whether the target is close enough to the current coverage pattern that allowable changes in the beam divergence and power would capture the target within an expanded coverage area. If this is so, then the OCS makes the necessary changes and beam acquisition can proceed. If not, the control algorithm determines if there is another setting of the switches that produces a coverage pattern that potentially includes the target. If such a setting exists, the OCS sets the necessary switches and adjusts the beam parameters to capture the target within the coverage area of the transmitter. The exact details of the switch settings depend on the combination of switches and couplers used in the transmitter design. For the simplest case shown in Figure 5, the control system only has to choose from two possible switch positions. There is necessarily a trade-off in the "Hybrid" design, as more switches reduce the power splitting losses of the couplers but increase the complexity of the control process If the target is outside of the potential coverage area for any switch combination, then control actions are requested from the BCS. A flowchart of this process is shown in Figure 9. 


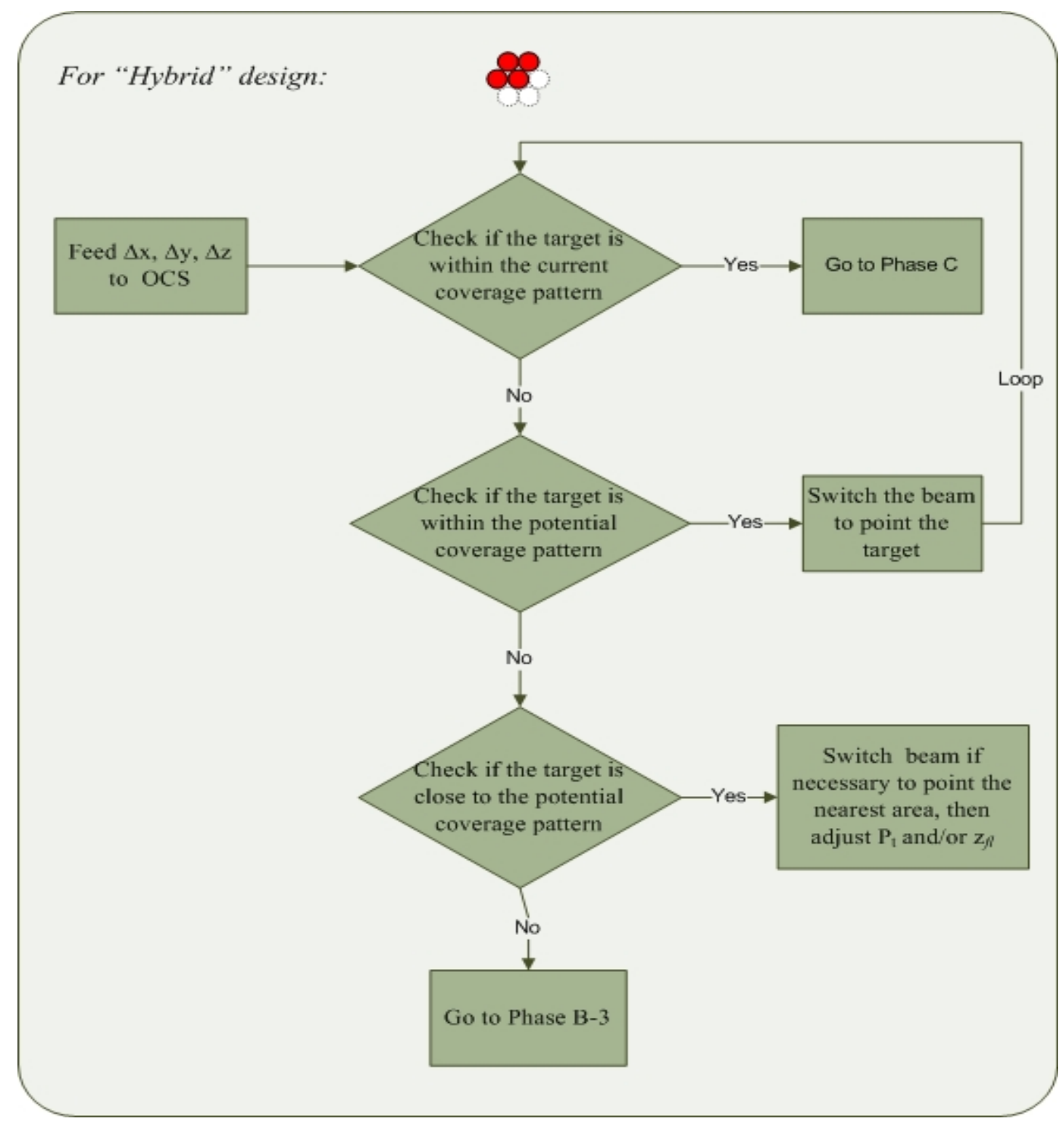

Figure 9. Control Flow for "Hybrid" Design

\section{Initial Evaluation}

The initial evaluation of the control process was performed theoretically by projecting its performance in a generic scenario. For this scenario, a unidirectional link was assumed, where one node was attempting to transfer data to a target node. For initial assessment, the transmitting node was considered strongly tethered, so that any movement of the node was negligible with respect to the errors inherent to the GPS system. A GPS error of 3 meters was assumed. The target node was allowed to move, whether due its own propulsion or in response to wind forces acting on the node. The effects of atmospheric turbulence were not accounted for in the initial assessment. At this stage, several key parameters impacting the success of the control system have been identified.

One key parameter is the rate at which the nodes update their GPS data with adjacent nodes in the network. This directly impacts the initial success of the BCS in effectively aligning the transmitter optics toward the receiver. Depending on the timing of a link request and the velocity of the target's motion, it is 
quite possible that transmitter will be directed such that no connection between the transmitter and target is possible, even if both nodes are functioning properly. A long delay between updates also adversely impacts attempts to track the target node, making control difficult and the link unreliable under all except the best of operating conditions, regardless of the speed of the controller.

A second key parameter is the error inherent in each of the positioning and data systems with which the control system interacts. A primary source of error is the accuracy of the GPS data. The 3-meter error assumed is quite significant for FSO links of even relatively short distances, even with the larger coverage areas available with advanced receiver designs. Additional sources of error include the accuracy of the BCS (determined by the control algorithm and the limitations of the mechanisms used to move the platform) and smaller error sources in the optical systems under the OCS jurisdiction. Even if the BCS and OCS are able to point the transmitter exactly at where the target is thought to be, the cumulative errors can cause the target to be missed.

The implication is that an additional control sequence must be added to the proposed algorithm to allow the control systems to perform some sort of search for the target node. The control calculations must be amended to account for the range of target locations, as dictated by the error expected, rather than assume that the target location is uniquely defined by the GPS data. The details of the search and the control level at which it is implemented depend on what search options are selected. The most direct search method would involve larger movement of the platform through the existing control options, with the BCS as the primary director. An alternate approach is to have the optical components mounted below the platform with a separate pointing control such as a gimbal or motor. Whether this search is controlled by the OCS or by the BCS depends on where the search process is situated within the overall control algorithm and whether it is coupled with a beam power and/or divergence adjustment.

Accounting for these key parameters requires significant adjustments to the nature of the proposed control algorithm. These adjustments are in the process of being implemented, and a more rigorous, simulation based assessment of the algorithm is planned once these adjustments are completed.

\section{Summary}

A control algorithm is proposed and evaluated for accomplishing acquisition and tracking in an FSO link. The algorithm assumes the existence of a master control system for realizing course alignment of the mobile platform and a separate control system for adjusting beam parameters and pointing. Several factors influence control decisions, including the transmitter design implemented at the node, the likelihood of completing the link with or without minor adjustments in the transmitter power and beam divergence, foreknowledge of the receiver design, data refresh rates, and errors in the positioning and data systems. Control actions at the fine optical level or the course mechanical level are then dictated and acted upon. Initial evaluation of the design is performed and indicates refinements in design and performance required to develop a practical control system.

\section{References}

[1] Dodley, J.P., D.M. Britz, D.J. Bowen, C.W. Lundgren, 2001, Free space optical technology and distribution architecture for broadband metro and local services, Proc. SPIE, 4214, pp. 72-85.

[2] Arnon, S., 2003, Optimization of urban optical wireless communication systems, IEEE Transactions on Wireless Communications 2, 626-629.

[3] Arnon, S., S. Rotman, N.S. Kopeika, 1997, Beam width and transmitter power adaptive to tracking system performance for free-space optical communication, Applied Optics 6, 6095-6101.

[4] Leitgeb, E., J. Bregenzer, M. Gebhart, P. Fasser, A. Merdonig, 2003, Free space optics - broadband wireless supplement to fiber networks, in Free-Space Laser Communication Technologies XV, G. S. Mecherle, Ed., Proc. SPIE 4975, pp. 57-68.

[5] Davis, C. C., I. I. Smolyaninov, S. D. Milner, 2003, Flexible optical wireless links and networks, IEEE Communications Magazine, pp. 51-57.

[6] Izadpanah, H., T. Elbatt, V. Kukshya, F. dolezal, B. K. Ryu, 2003, High availability free space optical and RF hybrid wireless networks, IEEE Wireless Communications, pp. 45-53.

[7] Zhou, D., P. G. LoPresti, N. Brooks, H. Refai, Evaluation of free-space optical fiber bundle transmitter configurations for receiver tracking, 2009, 
Proc. SPIE, Atmospheric Propagation VI, vol. 7324, pp. $73240 \mathrm{~K}-1$.

\section{Acknowledgements}

This work was supported by NSF Grant \#ECCS0725801

$28^{\text {th }}$ Digital Avionics Systems Conference

October 25 - 29, 2009 
6.A.3-12 